\title{
Strain Hardening of Fractal Colloidal Gels
}

\author{
T. Gisler, ${ }^{1, *}$ R. C. Ball, ${ }^{2}$ and D. A. Weitz ${ }^{1}$ \\ ${ }^{1}$ University of Pennsylvania, Department of Physics, 209 S 33rd Street, Philadelphia, Pennsylvania 19104 \\ ${ }^{2}$ University of Warwick, Department of Physics, Coventry, Warwickshire CV4 7AL, England
}

(Received 12 June 1998)

\begin{abstract}
We report on experiments on the rheology of gels formed by diffusion-limited aggregation of neutrally buoyant colloidal particles. These gels form very weak solids, with the elastic modulus, $G^{\prime}(\omega)$, larger than the loss modulus, $G^{\prime \prime}(\omega)$, and with both $G^{\prime}(\omega)$ and $G^{\prime \prime}(\omega)$ exhibiting only a very weak frequency dependence. Upon small but finite strains $\gamma<0.45$ the elastic modulus increases roughly exponentially with $\gamma^{2}$. We explain the observed strain hardening with the highly nonlinear elastic response of the rigid backbone of the gel to elongational deformation.

PACS numbers: 81.40.Jj, 82.70.Dd, 82.70.Gg, 83.50.Gd
\end{abstract}

Colloidal particles aggregating by attractive interactions form highly disordered clusters; these structures are, on average, self-similar, and the mass of a cluster, $M$, scales with its radius, $R$, as $M(R) \sim M_{0}(R / a)^{d_{\mathrm{f}}}$, where $a$ is the size of the colloidal monomer and $M_{0}$ is its mass [1]. The fractal mass exponent $d_{\mathrm{f}}$ characterizes the ramification of the cluster and varies between $d_{\mathrm{f}}=2.1$ for reactionlimited cluster aggregation and $d_{\mathrm{f}}=1.8$ for diffusionlimited cluster aggregation (DLCA) [2]. In the latter case the distribution of cluster sizes is fairly narrow and the characteristic cluster size grows linearly with time. Aggregation of clusters eventually leads to a spacefilling structure which is no longer fractal on all length scales but which can instead show long-range correlations as revealed by a scattering peak corresponding to a characteristic cluster size $R_{\mathrm{c}}=a \varphi^{1 /\left(d_{\mathrm{f}}-3\right)}$, where $\varphi$ is the initial volume fraction of monomer particles [3,4]. The clusters themselves are close packed, forming a ramified, tenuous gel structure. This structure should be an elastic material with unique properties, which are determined not only by $d_{\mathrm{f}}$, but also by the connectivity or chemical dimension, $d_{\mathrm{b}}$, which characterizes the scaling of the contour length within the cluster. The elasticity of such a tenuous structure can be expected to be extremely weak; however, it should increase dramatically with increasing $\varphi$. Since the backbone of the gel is contorted, a shear strain will be accommodated by a lengthening of the backbone, stretching out the inherent bends. This should lead to highly unusual elastic properties; the stretching of the backbone will increase its rigidity and thus the gel can be expected to exhibit pronounced strain hardening, becoming increasingly stiffer as it is stretched, until it catastrophically breaks. However, because of the very weak elasticity and the difficulty of working with completely unsheared, virgin gels, this interesting behavior has never been observed.

By contrast, strain hardening has been observed in other types of gels; rubbers and swollen polymer gels exhibit strain hardening when they are stretched enough that the chains are no longer described by Gaussian statistics $[5,6]$.
Strain hardening also occurs in a completely different class of materials, semiflexible biopolymers such as cellulose [7], F-actin [8], and vimentin [9]. Such polymers are responsible for the mechanical stability of biological tissues; however, the origin of the strain hardening in these systems remains obscure. Although structurally different, colloidal aggregate gels share an essential feature with biopolymer networks: locally they each consist of segments with significant bending rigidity, which dominates their elastic properties. Thus, an understanding of the elasticity and strain hardening of colloidal gels may also provide important insight into the properties of these biopolymer networks.

In this paper, we present measurements of the elastic properties of virgin colloidal aggregate gels. We show that their very weak elasticity increases as $\varphi^{3.2}$, in agreement with theoretical expectations for a fractal network. In addition, we also show that the gels do indeed exhibit a pronounced strain hardening before they catastrophically break. Surprisingly, the data can all be scaled onto a single master curve, independent of $\varphi$. We quantitatively account for this strain hardening and the observed scaling using a model that predicts the nonlinear stress response of a network of randomly oriented backbones of connected clusters.

We use polystyrene latex particles with diameter $2 a=$ $19 \mathrm{~nm}$ in a neutrally buoyant mixture of $\mathrm{H}_{2} \mathrm{O}$ and $\mathrm{D}_{2} \mathrm{O}$ $[3,10]$ at $25^{\circ} \mathrm{C}$; adjusting the density of the solvent to the particle density is necessary to suppress sedimentation which leads to either compactification of clusters and more rigid structures (work hardening), or, at larger stresses, breakage of bonds before clusters touch each other (brittle regime) [11]. We initiate aggregation by adding $\mathrm{MgCl}_{2}$ to a concentration of $6 \mathrm{mM}$; with initial volume fractions $4.6 \times 10^{-3} \leq \varphi \leq 1.58 \times 10^{-2}$, gels form within several hours, leading to macroscopically homogeneous structures. Static light scattering $[4,10,12]$ from these gels at lower $\varphi$ confirms $d_{\mathrm{f}} \sim 1.9$, slightly above the value expected for DLCA, and consistent with the initial aggregation being in the intermediate regime between 
diffusion- and reaction-limited cluster aggregation; we note, however, that diffusion still dominates at longer times, leading to a well-defined average cluster size.

We measure the linear storage and loss moduli $G^{\prime}$ and $G^{\prime \prime}$ with a controlled-strain rheometer in an oscillatory shear experiment using a double-wall Couette geometry. The gels are allowed to fully form in the rheometer cell, ensuring that we measure the properties of the undisturbed gel. Typical frequency-dependent moduli $G^{\prime}$ and $G^{\prime \prime}$ are shown in Fig. 1, for a gel with $\varphi=8.9 \times 10^{-3}$, and using a strain amplitude $\gamma=8 \times 10^{-3}$; reducing $\gamma$ yielded the same values of $G^{\prime}(\omega)$ and $G^{\prime \prime}(\omega)$, confirming linearity. The storage modulus is about an order of magnitude larger than the loss modulus and is only very weakly dependent on frequency; this is consistent with the formation of a solid gel structure. The loss modulus is also nearly independent of frequency, except at the very lowest frequencies probed, where a small increase indicates persistent loss mechanisms presumably indicative of very slow rearrangements within the gel; we emphasize, however, that these rearrangements occur on a time scale that is many orders of magnitude slower than the measurement time.

The dependence of $G^{\prime}$ on $\varphi$ is shown in Fig. 2. We find a power-law scaling $G^{\prime}(\varphi) \sim \varphi^{\nu}$, comparable with measurements of the compactive strength [13]. The magnitude of the modulus reflects the fact that the elasticity is governed by the intrinsic stiffness of the colloidal aggregate network. By contrast, a polymer gel, whose elasticity is entropic in origin, would have $G^{\prime} \sim N k_{\mathrm{B}} T$, where $N$ is the cross-link density; since $N=1 / R_{\mathrm{c}}^{3}$, these colloidal gels have a modulus that is roughly 4 orders of magnitude larger than an equivalent entropic gel. To account for the observed $\varphi$ dependence, we assume that the modulus is determined by the spring constant of the backbone

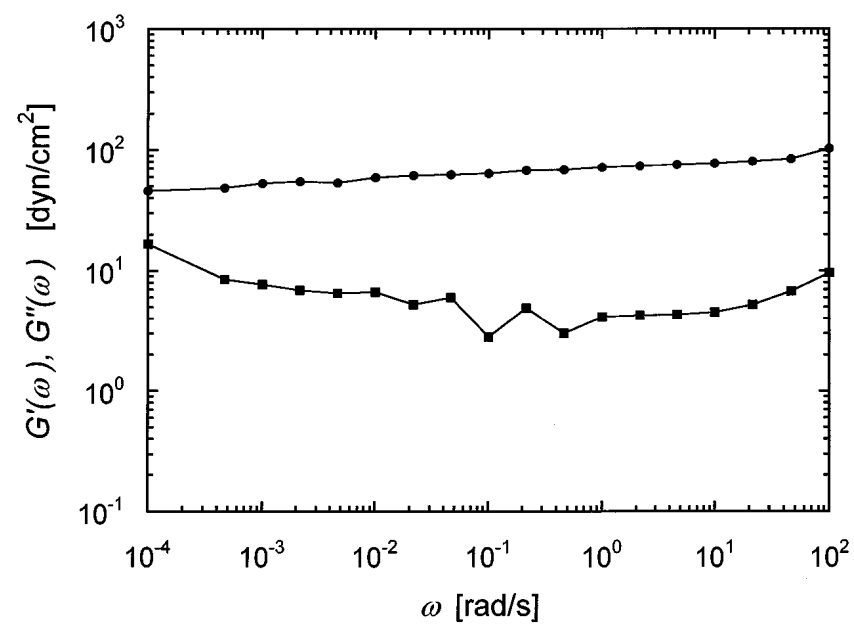

FIG. 1. Linear storage modulus $G^{\prime}(\omega)(0)$ and loss modulus $G^{\prime \prime}(\omega)(\boldsymbol{\square})$ as a function of angular frequency $\omega$ at volume fraction $\varphi=8.9 \times 10^{-3}$ after full gelation of clusters. The strain amplitude of the oscillatory shear was $\gamma=8 \times 10^{-3}$. of the average cluster. The spring constant of a fractal is size dependent, and is given by $k(R)=k_{0}(R / a)^{-\left(2+d_{\mathrm{b}}\right)}$ [14], where $k_{0}$ is the bending constant of an elementary bond. This expression reflects the fact that the extension of a fractal cluster occurs through the unbending of the contorted path of the chain; thus the spring constant decreases as the cluster size increases and contortions become easier to unbend. The spring constant depends inversely on the cross-sectional area of the chains, assumed to be $R^{2}$, and inversely on the longitudinal chemical length, which is proportional to $R^{d_{\mathrm{b}}}$. The elastic modulus of the gel is then $G^{\prime}=k\left(R_{\mathrm{c}}\right) / R_{\mathrm{c}}$, yielding an exponent of $\nu=\left(3+d_{\mathrm{b}}\right) /\left(3-d_{\mathrm{f}}\right)$ [15]. Experimentally, we find $\nu=3.2 \pm 0.3$; given the sensitivity of $\nu$ to $d_{\mathrm{f}}$, this is in excellent agreement with the predicted value, which is between 3.4 and 4.1 for $d_{\mathrm{f}}$ between 1.8 and 2, and using $d_{\mathrm{b}}=1.1$, obtained from computer simulations [16]. In addition, at the lowest volume fractions, the magnitude of $G^{\prime}$ agrees very well with the value determined from dynamic light scattering [10].

The fractal structure and connectivity suggests another important consequence for the elastic properties of the gel; as the network is strained, and the inherent bends are removed, we expect a rapid increase in the elastic modulus with increasing strain. We test for this using a series of gels of different $\varphi$ by measuring the dependence of $G^{\prime}$ and $G^{\prime \prime}$ on the maximum strain $\gamma$ of an oscillatory measurement at a fixed frequency of $10 \mathrm{rad} / \mathrm{s}$. As shown in Fig. 3, we observe a linear response for $\gamma<0.1$, where both $G^{\prime}$ and $G^{\prime \prime}$ are independent of $\gamma$. Further increase in strain amplitude results in a very rapid increase in $G^{\prime}$ and $G^{\prime \prime}$, up to $\gamma \approx 0.45$ above which both moduli drop precipitously; larger strains are no longer recoverable, indicating irreversible breakage of bonds between clusters. The behavior of these gels is in strong contrast to the marked shear thinning observed in weakly flocculated suspensions where both $G^{\prime}$ and $G^{\prime \prime}$ decrease above a yield strain which is strongly dependent on $\varphi$ [17].

To account for the strain hardening, we consider the effects of a large strain on a single, stress-bearing segment of the network and average the contributions from randomly oriented segments. In a randomly connected network, shear stress is accommodated by a longitudinal stretching of the strands that form the backbone. A large longitudinal strain, $\lambda$, stretches an individual strand, pulling out the bends that govern its elasticity, and making it anisotropic in shape by increasing its length while decreasing its width. This drastically modifies its elastic constant, which is now characterized in terms of the new width, $\xi$. Expressing the strain as $\lambda=g \xi / R_{\mathrm{c}}$, where $g=\left(R_{\mathrm{c}} / \xi\right)^{d_{\mathrm{b}}}$ is the number of blobs of size $\xi$, the new width is $\xi=R_{\mathrm{c}} \lambda^{1 /\left(1-d_{\mathrm{b}}\right)}$. Then, the spring constant of the extended strand is given by that of $g$ springs in a series, yielding $k\left(\lambda R_{\mathrm{c}}\right)=k_{0}\left(R_{\mathrm{c}} / a\right)^{-\left(2+d_{\mathrm{b}}\right)} \lambda^{2 /\left(d_{\mathrm{b}}-1\right)}$. This spring constant is very sensitive to the strain, $\lambda$, for typical values of $d_{\mathrm{b}}$, which are close to unity [16]. The force 


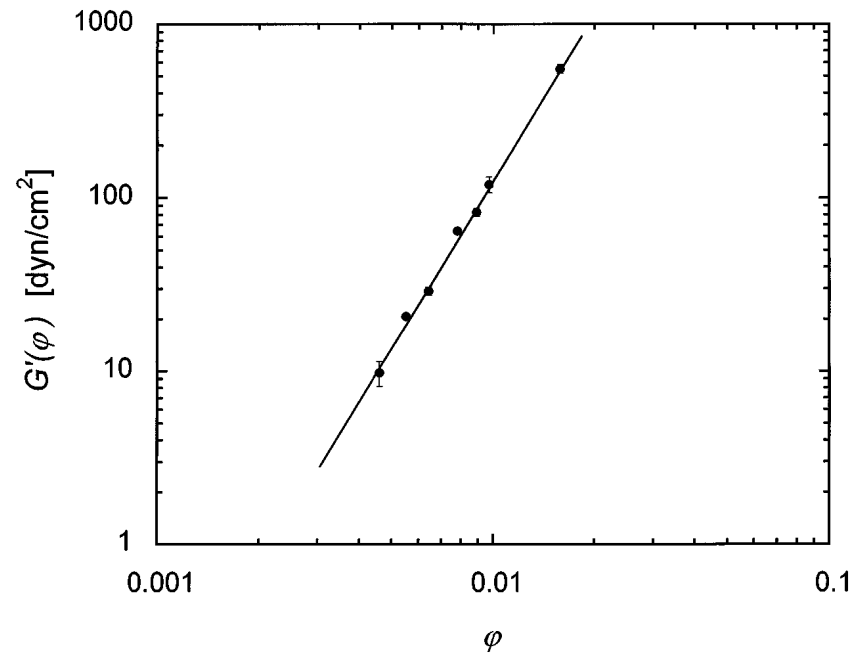

FIG. 2. Elastic modulus $G^{\prime}$ of gelled polystyrene latex as a function of volume fraction $\varphi$ of primary particles, measured at small strain amplitudes $8 \times 10^{-4} \leq \gamma \leq 8 \times 10^{-3}$. The scaling $G^{\prime} \sim \varphi^{\nu}$ (full line), with an exponent $\nu \approx 3.2$, is consistent with the prediction $\nu=\left(3+d_{\mathrm{b}}\right) /\left(3-d_{\mathrm{f}}\right)=3.73$ using $d_{\mathrm{f}}=1.9$ and a value for the backbone dimension $d_{\mathrm{b}}=$ 1.1 for diffusion-limited aggregation.

required to stretch a backbone segment by a factor $\lambda$ is

$$
f(\lambda)=k_{0} a\left(R_{\mathrm{c}} / a\right)^{-1-d_{\mathrm{b}}}\left[\lambda^{A}-1\right],
$$

where $A=\left(1+d_{\mathrm{b}}\right) /\left(d_{\mathrm{b}}-1\right)$.

The strain dependence of this force leads to the strain hardening of the modulus. To calculate this, we assume that the deformation is affine, characterized by the deformation tensor $\Lambda$. Backbone strands are then both stretched and rotated by $\Lambda$, and the magnitude of $\lambda$ for any chain depends on its orientation $\mathbf{n}$ with respect to the principal axes of the deformation tensor, $\lambda=|\Lambda \mathbf{n}|$. The force along a strand is $f(|\Lambda \mathbf{n}|) \Lambda \mathbf{n} /|\Lambda \mathbf{n}|$, from which the total stress is calculated as

$$
\sigma=\frac{1}{R_{\mathrm{c}}^{2}} \int d^{2} r \Lambda \mathbf{n} \frac{\Lambda \mathbf{n}}{|\Lambda \mathbf{n}|} f(|\Lambda \mathbf{n}|),
$$

where we have assumed a uniform distribution of strand orientations. When $d_{\mathrm{b}} \approx 1$, extensional stresses dominate over contributions from compression and rotation; the force along a direction $\Lambda \mathbf{n}$ may then be approximated as $\mathbf{n} f(|\Lambda \mathbf{n}|)$, resulting in

$$
\sigma_{x y}=\frac{1}{R_{\mathrm{c}}^{2}} \iint \sin \theta d \theta d \phi \sin ^{2} \theta \sin 2 \phi f(\lambda(\theta, \phi)) .
$$

In the case of a simple shear along the $x$ axis with a strain $\gamma$ the extension ratio is $\lambda(\theta, \phi)=(1+$ $\left.\gamma \sin ^{2} \theta \sin 2 \phi+\gamma^{2} \sin ^{2} \theta \cos ^{2} \phi\right)^{1 / 2}$ and the force becomes

$$
\begin{aligned}
f(\lambda)=L[1 & +\gamma \sin ^{2} \theta \sin 2 \phi \\
& \left.+\gamma^{2} \sin ^{2} \theta \cos ^{2} \phi\right]^{A / 2}-L,
\end{aligned}
$$

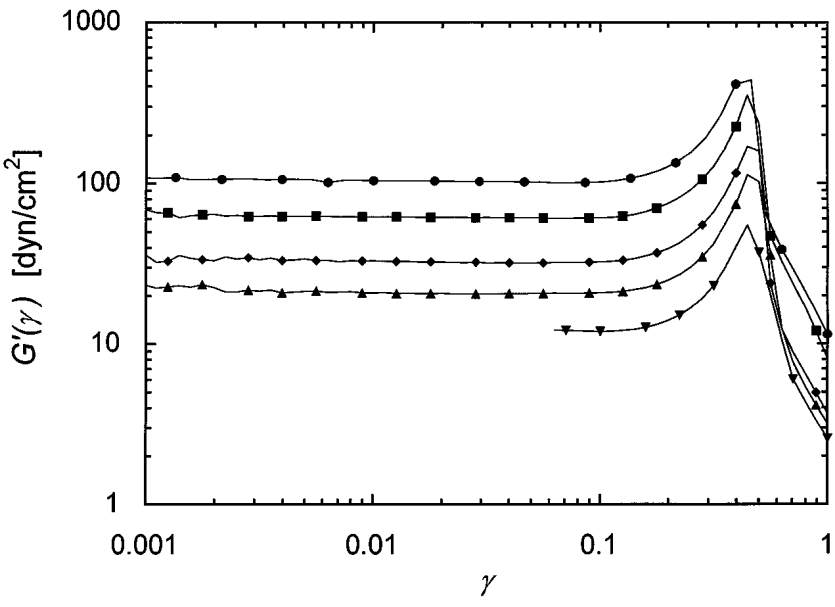

FIG. 3. Elastic modulus $G^{\prime}$ as a function of the strain amplitude $\gamma$ in an oscillatory shear experiment. The volume fractions are $\varphi=9.7 \times 10^{-3}(\boldsymbol{O}), \varphi=7.8 \times 10^{-3}(\mathbf{\square})$, $\varphi=6.4 \times 10^{-3}(\boldsymbol{\bullet}), \quad \varphi=5.5 \times 10^{-3}(\mathbf{\Delta}), \quad$ and $\varphi=$ $4.6 \times 10^{-3}(\boldsymbol{\nabla})$. The excitation frequency is $10 \mathrm{rad} / \mathrm{s}$. Strains beyond $\gamma \approx 0.45$ result in (irreversible) breakage of the backbone.

$$
\sim L \exp \left[\frac{A \gamma}{2} \sin ^{2} \theta \sin 2 \phi+\frac{A \gamma^{2}}{2} \sin ^{2} \theta \cos ^{2} \phi\right]-L,
$$

where $L=k_{0} a\left(R_{\mathrm{c}} / a\right)^{-1-d_{\mathrm{b}}}$. For large values of $A\left(d_{\mathrm{b}} \approx\right.$ $1)$, there is a regime where terms quadratic in $\gamma^{2}$ are small. We neglect the quadratic term, integrate over all orientations with Eq. (3) and expand the exponential terms; then using $G^{\prime}=\sigma_{x y} / \gamma$ we obtain

$$
G^{\prime}(\gamma)=\frac{L A}{R_{\mathrm{c}}^{2}} \sum_{n=0}^{\infty} \frac{1}{(2 n+1) !} I_{4 n+5} I_{2 n+2}\left(\frac{A \gamma}{2}\right)^{2 n},
$$

where $I_{k}=\int_{0}^{2 \pi} \mathrm{d} \theta \sin ^{k} \theta$.

This result has an important consequence; Eq. (6) suggests that scaling individual $G^{\prime}(\gamma)$ curves at different volume fractions with their value at zero strain should yield a master curve for the excess elastic modulus that is independent of $\varphi$. We test this prediction in Fig. 4, where we plot all our data, rescaled by the modulus at small strain amplitudes, as a function of $\gamma$; the data do indeed follow the predicted scaling. Moreover, as shown by the solid curve, we obtain excellent agreement with the prediction Eq. (6) for $G^{\prime}(\gamma)$ for all $\varphi$ if we adjust the backbone dimension $d_{\mathrm{b}}$ to 1.07 , a value which is consistent with the scaling of the zero-strain elastic modulus with volume fraction. This value is also in remarkably good agreement with the results of computer simulations [16], particularly given the extreme sensitivity of the fit to $d_{\mathrm{b}}$.

Surprisingly, the stress response in the oscillatory shear experiments is remarkably linear, as measured by small third-harmonic stress components; however, we observe that the strains are not recoverable. This suggests 


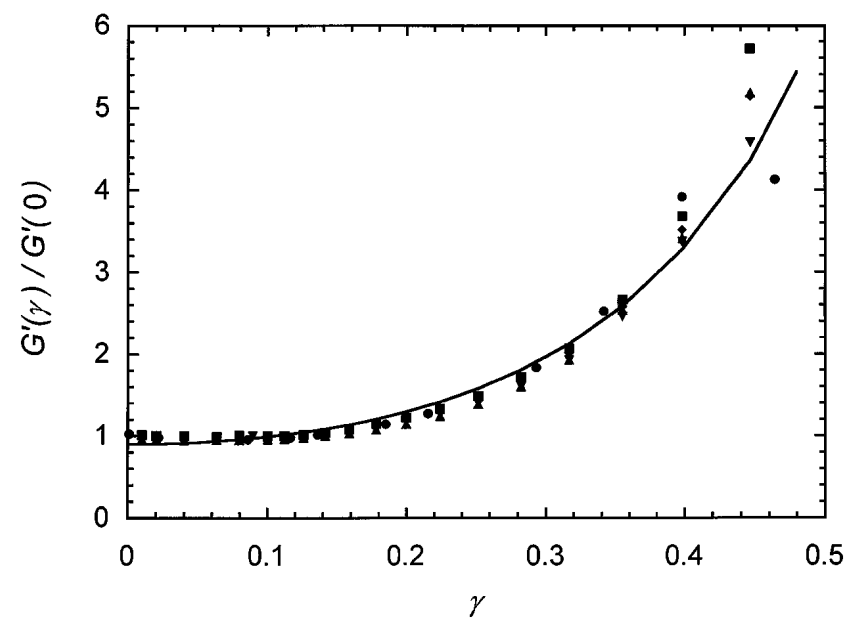

FIG. 4. Elastic modulus $G^{\prime}(\gamma)$ scaled with the zero-strain modulus $G^{\prime}(0)$ as a function of strain amplitude $\gamma$. Symbols denote different volume fractions of monomers (see Fig. 3). The full line shows a fit of a fourth-order power series in $\gamma^{2}$, using the theoretical expansion factors [see Eq. (6)].

that small but finite deformations lead to formation of contacts between side chains which now become part of the stress-conducting network; as the strain is released, these newly formed backbone segments either buckle or deform; deformation, however, counteracts the complete recoil of stretched segments belonging to the original network, leading to an enhanced modulus at zero extension. Upon further extension, the stress response is now mainly due to the first-generation backbone segments since their extension is large. This is consistent with the observation that the observed scaling of $G^{\prime}(\gamma)$ with $\gamma$ is somewhat steeper than we would expect using the predicted expansion coefficients.

The importance of buckling events is also revealed by the loss modulus: at fixed strain amplitude, $G^{\prime \prime}(\omega)$ is almost independent of frequency, suggesting that energy dissipation arises from displacements rather than from coupling to velocities that would depend on strain rates (i.e., $\gamma \omega$ in an oscillatory experiment). Then during each oscillation cycle, a constant fraction of the work stored in the deformed network is lost in conformational changes occurring locally on the length scale of a network segment, leading to a constant loss tangent $G^{\prime \prime} / G^{\prime}$; indeed, we also find that the loss tangent is practically independent of the strain amplitude.

Unlike the nonlinear elasticity of rubbers which is governed by entropic restoring forces [5], elasticity and strain hardening in fractal colloidal gels have their origin in the intrinsic stiffness of contorted backbone segments; increasing strain straightens the segments, resulting in an increase in the elastic modulus. Elasticity and strain hardening in fractal colloidal gels rely critically on the continuous network of permanent bonds. The mechanism for strain hardening presented here might be useful for the understanding of the nonlinear rheology of semiflexible polymer networks; it certainly provides an excellent account of the behavior of colloidal gels.

We gratefully acknowledge support from NSF (DMR96-31279) and NASA (NAG3-2058); T.G. was also supported by the Schweizerischer Nationalfonds.

*Present address: Universität Konstanz, Fakultät für Physik, P.O. Box 5560, 78457 Konstanz, Germany.

[1] D. A. Weitz and M. Oliveria, Phys. Rev. Lett. 52, 1433 (1984).

[2] D. A. Weitz, J. S. Huang, M. Y. Lin, and J. Sung, Phys. Rev. Lett. 54, 1416 (1985).

[3] M. Carpineti and M. Giglio, Phys. Rev. Lett. 68, 3327 (1992).

[4] M. Carpineti and M. Giglio, Phys. Rev. Lett. 70, 3828 (1993).

[5] L. R. G. Treloar, The Physics of Rubber Elasticity (Clarendon Press, Oxford, 1975), 3rd ed.

[6] J. Baselga, I. Hernández-Fuentes, I. F. Piérola, and M. A. Llorente, Macromolecules 20, 3060 (1987).

[7] R. D. Groot, A. Bot, and W. G. M. Agterof, J. Chem. Phys. 104, 9202 (1996).

[8] P. A. Janmey, S. Hvidt, J. Käs, D. Lerche, A. Maggs, E. Sackmann, M. Schliwa, and T.P. Stossel, J. Biol. Chem. 269, 32503 (1994).

[9] P. A. Janmey, U. Euteneuer, P. Traub, and M. Schliwa, J. Cell Biol. 113, 155 (1991).

[10] A.H. Krall and D. A. Weitz, Phys. Rev. Lett. 80, 778 (1998).

[11] R. Wessel and R. C. Ball, Phys. Rev. A 46, R3008 (1992).

[12] D. Asnaghi, M. Carpineti, M. Giglio, and M. Sozzi, Phys. Rev. A 45, 1018 (1992).

[13] R. Buscall, P.D. A. Mills, J. W. Goodwin, and D. W. Lawson, J. Chem. Soc. Faraday Trans. 84, 4249 (1988).

[14] Y. Kantor and I. Webman, Phys. Rev. Lett. 52, 1891 (1984).

[15] R. C. Ball, Physica (Amsterdam) 38D, 13 (1989).

[16] P. Meakin, I. Majid, S. Havlin, and H. E. Stanley, J. Phys. A 17, L975 (1984).

[17] R. de Rooij, D. van den Ende, M.H.G. Duits, and J. Mellema, Phys. Rev. E 49, 3038 (1994). 